\title{
Immunohistochemical detection of virus through its nuclear cytopathic effect in idiopathic interstitial pneumonia other than acute exacerbation
}

\author{
G.C. dos Santos ${ }^{1}$, E.R. Parra ${ }^{1}$, F.W. Stegun ${ }^{2}$, C.S. Cirqueira ${ }^{1}$ and V.L. Capelozzi ${ }^{1}$ \\ ${ }^{1}$ Disciplina de Pneumologia, Departamento de Patologia, Faculdade de Medicina, Universidade de São Paulo, \\ São Paulo, SP, Brasil \\ ${ }^{2}$ Disciplina de Moléstias Infecciosas, Departamento de Patologia, Faculdade de Medicina, Universidade de São Paulo, \\ São Paulo, SP, Brasil
}

\begin{abstract}
Idiopathic interstitial pneumonias include complex diseases that have a strong interaction between genetic makeup and environmental factors. However, in many cases, no infectious agent can be demonstrated, and these clinical diseases rapidly progress to death. Theoretically, idiopathic interstitial pneumonias could be caused by the Epstein-Barr virus, cytomegalovirus, adenovirus, hepatitis C virus, respiratory syncytial virus, and herpesvirus, which may be present in such small amounts or such configuration that routine histopathological analysis or viral culture techniques cannot detect them. To test the hypothesis that immunohistochemistry provides more accurate results than the mere histological demonstration of viral inclusions, this method was applied to 37 open lung biopsies obtained from patients with idiopathic interstitial pneumonias. As a result, immunohistochemistry detected measles virus and cytomegalovirus in diffuse alveolar damage-related histological patterns of acute exacerbation of idiopathic pulmonary fibrosis and nonspecific interstitial pneumonia in 38 and $10 \%$ of the cases, respectively. Alveolar epithelium infection by cytomegalovirus was observed in $25 \%$ of organizing pneumonia patterns. These findings were coincident with nuclear cytopathic effects but without demonstration of cytomegalovirus inclusions. These data indicate that diffuse alveolar damage-related cytomegalovirus or measles virus infections enhance lung injury, and a direct involvement of these viruses in diffuse alveolar damage-related histological patterns is likely. Immunohistochemistry was more sensitive than the histological demonstration of cytomegalovirus or measles virus inclusions. We concluded that all patients with diffuse alveolar damage-related histological patterns should be investigated for cytomegalovirus and measles virus using sensitive immunohistochemistry in conjunction with routine procedures.
\end{abstract}

Key words: Fibrosing interstitial pneumonia; Viral infections; Immunohistochemistry; Tissue microarray

\section{Introduction}

Idiopathic interstitial pneumonias (IIPs) are considered to be complex diseases with a strong interaction between genetic makeup and environmental factors (1-3). The major IIPs include idiopathic pulmonary fibrosis (IPF), nonspecific interstitial pneumonia (NSIP), acute interstitial pneumonia (AIP), and bronchiolitis obliterans organizing pneumonia (BOOP). They are characterized by abnormal repair and remodeling of lung parenchyma with prominent alveolar septal thickening by fibroblasts, chronic inflammatory cells, and extensive confluent alveolar lining cells. The clinical presentation and prognosis are variable.
Some patients remain stable or improve with treatment, but the majority evolve to end-stage fibrosis and eventually die from the disease or infection.

IIPs may have an acute or subacute presentation, or an acute exacerbation (AE) may occur in previously subclinical or unrecognized chronic IIPS $(4,5)$. Pathologically, AEs show a combination of an underlying fibrotic interstitial pneumonia and a superimposed form of acute lung injury, either diffuse alveolar damage (DAD) or organizing pneumonia (OP) (6). Numerous enlarged alveolar lining cells with significant nuclear cytopathic

Correspondence: V.L. Capelozzi and/or E.R. Parra, Departamento de Patologia, Faculdade de Medicina, USP, Av. Dr. Arnaldo, 455, Sala 1143, 01246-903 São Paulo, SP, Brasil. Fax: +55-11-3064-2744. E-mail: vcapelozzi@lim05.fm.usp.br and/or erparra20003@ yahoo.com.br 
effect (NCE) - characterized by cytologic atypia, prominent nucleoli, and mitotic figures - are also present.

The etiology of $A E$ is not known, and most patients do not have obvious precipitating factors. By definition, patients with $A E$ do not have an easily diagnosable infectious process, but the common presentation of $A E$ with flu-like symptoms, fever, and lavage neutrophilia raises the question of an occult infection (5). It is possible that viral or other infections that would produce little response in the normal host are able to cause DAD or OP in these highly abnormal lungs. Attempts to demonstrate specific pathogens have been unsuccessful; in particular, no association has been shown between $A E$ and chlamydial infections, Epstein-Barr virus (EBV), human herpesvirus, or cytomegalovirus (CMV) infections (7-12). The final diagnosis of viral infection is the result of the association between clinical and histological patterns.

The histological pattern in virus infection relies on morphological evidence of different degrees of lung injury affecting cellular pulmonary elements, resulting in interstitial pneumonia, currently associated with classical viral inclusion (CVI). Otherwise, when $\mathrm{CVI}$ is absent and a profound NCE is found in its place, the pathologist is confronted with the problem of establishing the definitive diagnosis of viral etiology. Thus, in many cases, no viral agent can be demonstrated. Hypothetically, IIPs could be caused by a virus, which, however, may be present in such small amounts or in such a configuration that mere routine histological analysis or viral culture cannot detect the virus. All of these factors are enhanced by the strategically multifocal or segmental distribution of viral infection in lungs, frequently not assessed even in surgical lung biopsies, thus limiting the diagnosis for the autopsy procedure. Recent advances in immunohistochemistry (IHC) with antibodies of type specificity confer great advantages in relation to diagnosing viral infection with regard to both sensitivity and specificity. In fact, using this technique, some investigators detected viral antigen also in cells with cytopathic effects, but without CVI (13).

To test the hypothesis that $\mathrm{IHC}$ techniques are more sensitive than histological demonstrations of virus inclusion, we performed an immunohistochemical study to detect viral antigens in AE of IPF and NSIP. To determine whether viral antigen expression was peculiar to $A E$ or a more generalized feature of fibrotic lung diseases, we also stained lung sections from patients with IPF, NSIP, AIP, and BOOP.

\section{Material and Methods}

\section{Diagnostic criteria}

Diagnosis of IPF, NSIP, AIP, and BOOP and their histological patterns was carried out according to the American Thoracic Society/European Respiratory Society consensus classification (1).

$\mathrm{AE}$ was diagnosed according to the criteria of Akira et al. (14): 1) subjective worsening of dyspnea within the past month; 2) new ground-glass opacities or consolidation on chest radiograph or high-resolution computed tomography (HRCT); 3) hypoxemia with decline $\geqslant 10 \mathrm{mmHg}$ in $\mathrm{PaO}_{2}$ from the previous level; 4) no evidence of infection by negative respiratory culture and serological test results for respiratory pathogens; 5) no clinical evidence of pulmonary embolism, congestive heart failure, or pneumothorax as a cause of acute worsening. However, 10 of the subjects did not have $\mathrm{PaO}_{2}$ data prior to $\mathrm{AE}$ because they first presented at the time of $A E$, and their surgical lung biopsy performed at that time showed DAD superimposed on the usual patterns of interstitial pneumonia (UIP; 5 cases) and NSIP (3 cases).

\section{Subjects}

From 1993 to April 2011, 37 consecutive patients with radiological diffuse infiltrates of the lungs as indicated by HRCT were submitted to an open lung biopsy at Hospital das Clínicas, Faculdade de Medicina, Universidade de São Paulo, according to the criteria outlined in the American Thoracic Society/European Respiratory Society international multidisciplinary consensus classification of IIPs (1). Among these patients, AE was diagnosed in five patients with UIP/IPF and in three patients with NSIP. All clinical and laboratory data were collected retrospectively from medical records. Pulmonary function tests included vital capacity, forced expiratory volume in $1 \mathrm{~s}$ (FEV1), forced vital capacity (FVC), FEV1/ FVC ratio $\times 100$, total lung capacity, residual volume, and carbon monoxide transfer factor (diffusing capacity of the lung for carbon monoxide). All HRCT were performed with 1.0- or 1.5-mm thick sections at supine and full inspiration at $10-\mathrm{mm}$ intervals. A specialized chest radiologist and a pneumologist analyzed the images at three preestablished levels (trachea, carina, and pulmonary veins) for the presence of any signs of diffuse parenchymal lung disease: ground glass, consolidation, reticular, honeycombing, and bronchiectasia. Biopsy slides were reviewed independently by at least two pathologists and reclassified according to the American Thoracic Society/ European Respiratory Society consensus classification of interstitial pneumonia as described in detail in an earlier report (1). After diagnosis by surgical lung biopsy, all the patients received corticosteroid. Negative CMV serology status was known for 11 patients, and no CMV prophylaxis was used. The acute deterioration in clinical course was demarcated by progressive respiratory failure in all 37 patients and allowed a pathological evaluation. Viral culture results were obtained after surgical lung biopsy and revealed no CMV, adenovirus (ADV), or respiratory syncytial virus (RSV) in 37 cases. Further details about these patients are summarized in Table 1. Among these, $49 \%(n=18)$ were males and $51 \%(n=19)$ females. The median age of the patients was 66 years (range 34-79) for 
Table 1. Clinical data of patients with idiopathic pulmonary fibrosis, nonspecific interstitial pneumonia, acute interstitial pneumonia, and bronchiolitis obliterans organizing pneumonia.

\begin{tabular}{|c|c|c|c|c|c|c|c|c|}
\hline \multirow{2}{*}{$\begin{array}{l}\text { Underlying } \\
\text { disease }\end{array}$} & \multirow{2}{*}{$\begin{array}{l}\text { Age (years)/ } \\
\text { gender }\end{array}$} & \multicolumn{3}{|c|}{ Clinical data } & \multirow[t]{2}{*}{ Pathological pattern } & \multirow[t]{2}{*}{ NCE } & \multirow[t]{2}{*}{$\mathrm{CVI}$} & \multirow[t]{2}{*}{ Virus $\mathrm{IHC}$} \\
\hline & & Dyspnea & Fever & $\begin{array}{l}\text { Tobacco } \\
\text { history }\end{array}$ & & & & \\
\hline IPF & $34 / F$ & + & - & + & UIP & + & - & none \\
\hline IPF & $52 / F$ & + & + & + & UIP & + & - & none \\
\hline IPF & $76 / F$ & + & - & + & UIP & + & - & none \\
\hline IPF & $69 / F$ & + & - & - & UIP & + & - & none \\
\hline IPF & $79 / F$ & + & - & - & UIP & + & - & none \\
\hline IPF & $74 / F$ & + & + & + & UIP plus DAD & + & - & MV \\
\hline IPF & $72 / \mathrm{M}$ & + & + & + & UIP plus DAD & + & - & none \\
\hline IPF & $70 / \mathrm{M}$ & + & + & + & UIP plus DAD & + & - & CMV \\
\hline IPF & $66 / \mathrm{M}$ & + & + & - & UIP plus DAD & + & - & none \\
\hline IPF & $66 / \mathrm{M}$ & + & + & - & UIP plus DAD & + & - & $\mathrm{MV}$ \\
\hline IPF & $66 / \mathrm{M}$ & + & - & + & UIP & + & - & none \\
\hline IPF & $71 / \mathrm{M}$ & + & - & - & UIP & + & - & none \\
\hline IPF & $66 / \mathrm{M}$ & + & - & - & UIP & + & - & none \\
\hline NSIP & $76 / F$ & + & - & - & NSIP & + & - & none \\
\hline NSIP & $65 / F$ & + & - & + & NSIP & + & - & none \\
\hline NSIP & $67 / F$ & + & - & + & NSIP & + & - & none \\
\hline NSIP & $47 / F$ & + & + & - & NSIP plus DAD & + & - & CMV \\
\hline NSIP & $65 / F$ & + & + & - & NSIP plus DAD & + & - & none \\
\hline NSIP & $65 / \mathrm{M}$ & + & + & - & NSIP plus DAD & + & - & none \\
\hline NSIP & $65 / \mathrm{M}$ & + & - & - & NSIP & + & - & none \\
\hline NSIP & $69 / \mathrm{M}$ & + & - & - & NSIP & + & - & none \\
\hline AIP & $40 / \mathrm{M}$ & + & + & + & DAD & + & - & CMV \\
\hline AIP & $42 / \mathrm{M}$ & + & + & - & DAD & + & - & CMV \\
\hline AIP & $52 / \mathrm{M}$ & + & + & - & DAD & + & - & $\mathrm{MV}$ \\
\hline AIP & $85 / F$ & + & + & - & DAD & + & - & MV \\
\hline AIP & $50 / F$ & + & + & - & DAD & + & - & $\mathrm{MV}$ \\
\hline AIP & $46 / F$ & + & + & - & DAD & + & - & none \\
\hline AIP & $40 / F$ & + & + & + & DAD & + & - & none \\
\hline AIP & $35 / F$ & + & + & + & DAD & + & - & none \\
\hline AIP & $49 / \mathrm{F}$ & + & + & - & DAD & + & - & none \\
\hline AIP & $49 / \mathrm{M}$ & + & + & - & DAD & + & - & none \\
\hline AIP & $57 / \mathrm{M}$ & + & + & - & DAD & + & - & none \\
\hline AIP & $49 / \mathrm{M}$ & + & + & + & DAD & + & - & none \\
\hline BOOP & $75 / F$ & + & + & + & $\mathrm{OP}$ & + & - & CMV \\
\hline BOOP & $72 / \mathrm{F}$ & + & + & + & OP & + & - & none \\
\hline BOOP & $70 / \mathrm{M}$ & + & + & - & OP & + & - & none \\
\hline BOOP & $72 / \mathrm{M}$ & + & + & - & OP & + & - & none \\
\hline
\end{tabular}

NCE: nuclear cytopathic effect; CVI: classical virus inclusion; IHC: immunohistochemistry; F: female; M: male; IPF: idiopathic pulmonary fibrosis; NSIP: nonspecific interstitial pneumonia; AIP: acute interstitial pneumonia; BOOP: bronchiolitis obliterans organizing pneumonia; UIP: usual interstitial pneumonia; DAD: diffuse alveolar damage; OP: organizing pneumonia; CMV: cytomegalovirus; MV: measles virus.

IPF (7 men, 6 women), 65 years (range $47-76)$ for NSIP (3 men, 5 women), 49 years (range 35-85) for AIP (6 men, 6 women), and 72 years (range 70-75) for BOOP (2 men, 2 women). All 37 patients signed a free informed consent form and the study was approved by the local Ethics Committee.

\section{Virus detection tests}

Tissue microarray (TMA). An average of four tissue cylinders (each $1 \mathrm{~mm}$ in diameter) per original paraffin block were removed from the different areas of UIP, acute exacerbation of UIP, NSIP, acute exacerbation of NSIP, DAD, and OP and carefully examined under high 


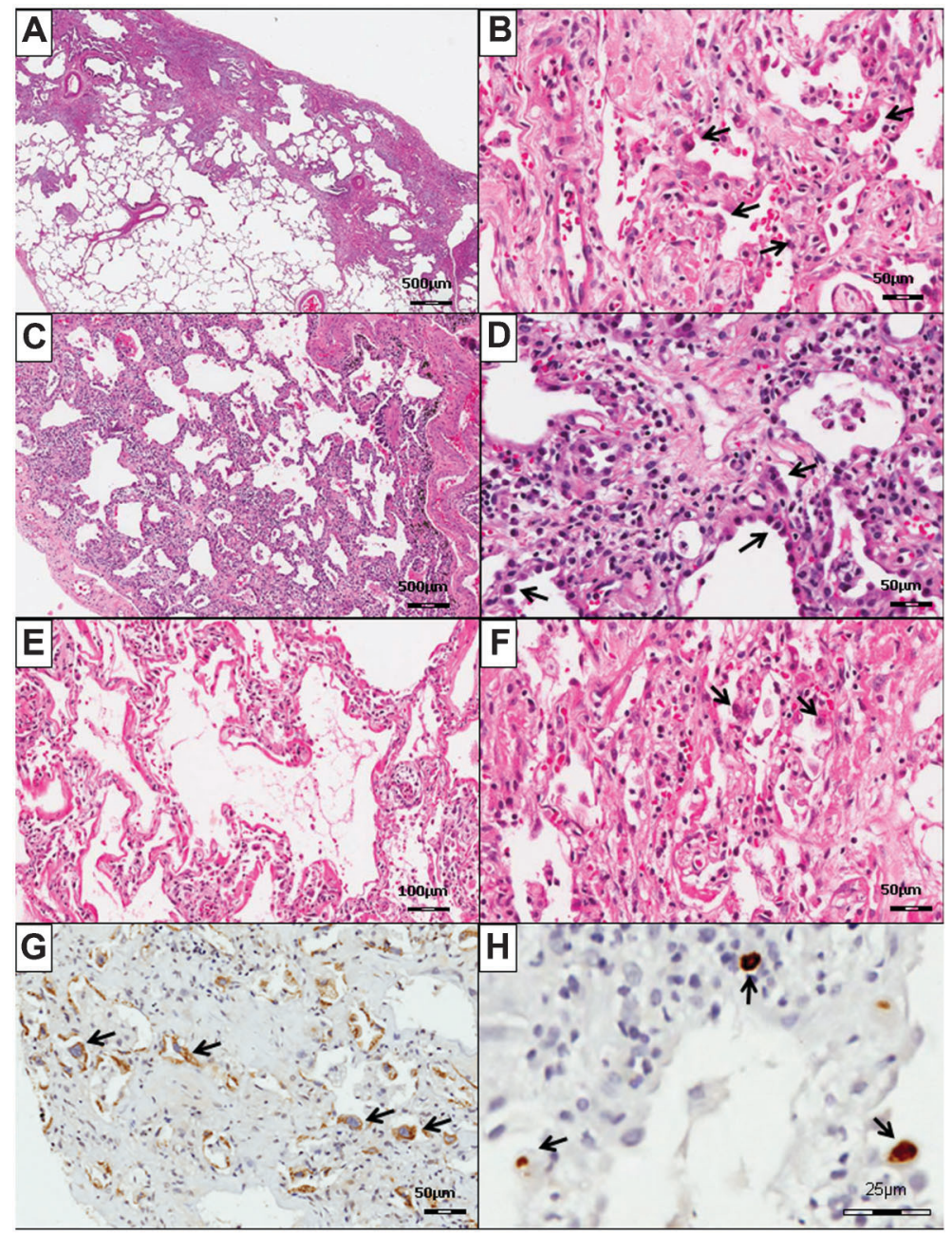

Figure 1. Panoramic view of the lung in usual interstitial pneumonia $(A)$, nonspecific interstitial pneumonia $(C)$ and diffuse alveolar damagerelated $(E)$ histological patterns and immunohistochemistry for measles virus (MV) $(G)$ and cytomegalovirus (CMV) $(H)$. At high magnification, note that prominent cuboidal hyperplasia of type II pneumocytes line the thickened interstitium in usual interstitial pneumonia $(B)$ and nonspecific interstitial pneumonia $(D)$. In panel $F$, diffuse alveolar damage was characterized by hyaline membranes and interstitial acute inflammation in a uniform temporal appearance present in diffuse distribution. In panels $B, D$ and $F$, the characteristics of the nuclear cytopathic effect (arrows) are shown. In panels $G$ and $H, M V$ and CMV intranuclear inclusion is visible after immunohistochemistry. magnification for cellular enlargement, intranuclear inclusions, ground-glass patterns, and multinucleated giant cells. These areas subsequently fitted into empty slots in recipient paraffin blocks (15). Two recipient blocks were created for the study, eventually containing 154 and 157 tissue cylinders, respectively. A total of six paraffinembedded sections per block were immunostained.

IHC. Sections from TMA paraffin blocks were used for IHC. The antibodies used were: anti-EBV 1:30 (M0897; Dako, USA), anti-CMV 1:20 (M0854; Dako), anti-ADV 1:1600 (MAB805; Chemicon, USA), anti-hepatitis C virus (HCV) 1:20 (18-7252; Zymed, USA), anti-RSV 1:400 (NCL-RSV-3; Novocastra, USA), anti-human herpesvirus1 (HHV-1) 1:1000 (B0114; Dako), anti-HHV-2 1:1000 (B0116; Dako), and anti-measles virus (MV) 1:100 (MAB8906; Millipore, USA). IHC was performed using the Envision (+) kit (Dako Cytomation, USA) according to the manufacturer's instructions. Sections were visualized by treating the slides with diamino-benzidine tetrahydrochloride. To demonstrate antibody specificity, sections from each paraffin block were used as negative controls by omitting the primary antibodies and replacing them with normal mouse or rabbit immunoglobulin. Positive expression of EBV, CMV, ADV, HCV, RSV, HHV-1, HHV-2, and MV were indicated by brown cytoplasmic or nuclear staining.

Histopathology. Virus detection was investigated histopathologically in IIPs, according to the following patterns: UIP, the histological pattern of IPF, where lung parenchyma shows architectural distortion with alternating areas of normal parenchyma, fibroblastic foci, marked fibrosis, honeycomb, and prominent cuboidal hyperplasia of type II pneumocytes lining the thickened interstitium (Figure 1A and B); NSIP, the histological pattern of NSIP, where lung parenchyma shows preserved lung architecture with uniform alveolar wall thickened by interstitial infiltrate composed of lymphocytes and plasma cells or dense interstitial fibrosis and cuboidal hyperplasia of pneumocytes (Figure $1 \mathrm{C}$ and $\mathrm{D}$ ); DAD, the histological pattern of AIP, 
where lung parenchyma shows diffuse alveolar wall thickening by proliferating connective tissue and inflammation, hyaline membranes, and prominent cuboidal hyperplasia of type II pneumocytes (Figure 1E and F); OP, the histological pattern of idiopathic centrilobular fibrosis, where lung parenchyma shows an airway-centered involvement by inflammation, cuboidal hyperplasia of type II pneumocytes, and fibrosis; AE, where lung parenchyma shows a combination of an underlying fibrotic interstitial pneumonia and superimposed DAD.

\section{NCE}

NCE was defined in type II pneumocytes by nuclear and cytoplasmic enlargement with hyperchromatic, atypical-appearing nuclei and abundant eosinophilic cytoplasm (Figure 1B, D, and F).

\section{CVIs}

Generally, CVIs were defined by a ground-glass nucleus with chromatin margination or an intranuclear Cowdry-type inclusion - an inclusion with or without intracytoplasmic inclusions. Specifically, EBV inclusions were identified as nuclear bodies in multinucleated giant cells. The intranuclear CMV inclusions were defined by central, dark-purple bodies that were separated from the surrounding chromatin by a clear halo; the cytoplasmic inclusions appear as coarse basophilic granules. ADV inclusions were defined by a homogeneous, amphophilic, or basophilic mass that almost completely filled the nucleus. HHV intranuclear inclusions were bright and red without cytoplasmic inclusions. MV was found in multinucleated giant cells containing eosinophilic intranuclear and intracytoplasmic inclusions. RSV was identified by small, round, eosinophilic intracytoplasmic inclusion bodies surrounded by a clear halo.

\section{Virus-inclusion evaluation}

The IHC procedure is a very sensitive method for determining the presence of virus, but it is true that there may be some confounding factors (cross-reactions with some components present in the tissue, particles present in the biopsy with the same appearance of the chromogen, etc.) that can produce fake results if not carefully evaluated. On account of this, we considered only positive reactions when the cells exhibited focal points of intense and vivid brown color without diffusely spread staining on pulmonary parenchyma. To determine the presence of virus by histological tests and IHC, microscopic fields were evaluated in each case at a magnification of $400 \times$, according to the extent of stained cells.

\section{Results}

Clinical, histopathological, and immunohistochemical data are shown in Table 1.
On histological examination, the lungs showed the most evident lesions. Thus, IIPs were histologically represented by UIP $(n=8 / 21 \%), A E$ of UIP $(n=5 / 13 \%)$, NSIP $(n=5 / 13 \%)$, AE of NSIP $(n=3 / 8 \%)$, DAD $(n=12 /$ $32 \%)$, and OP $(n=4 / 11 \%)$.

NCE was detected on type II pneumocytes in all cases of UIP, AE of UIP, NSIP, AE of NSIP, DAD, and BOOP. However, no CVI was detected in these histological patterns.

Cells that were IHC positive for CMV (Figure $1 \mathrm{H}$ ) were identified in type II pneumocytes and endothelial cells. CMV immunoreactivity was detected in DAD histological patterns found in AIP (2 cases), AE of IPF (1 case), and NSIP (1 case), and in 1 case with an OP histological pattern. These cases were without CVIs but showed an NCE. Owing to the random and sparse numbers of CMV inclusion-bearing cells in the section (Figure 1H), CMV infection of the lung parenchyma was scant. However, CMV infection was mainly responsible for severe DAD found in these patients.

Cells that were IHC positive for MV (Figure 1G) were recognized in type II pneumocytes. MV immunoreactivity was identified in DAD histological patterns of acute exacerbation of IPF ( 2 cases) and in AIP (3 cases). These five cases were without the CVIs but exhibited an NCE. Owing to widespread occurrence and many MV inclusion-bearing cells (Figure $1 \mathrm{G}$ ), MV infection of the lung was prominent and also responsible for severe DAD in these patients.

EBV, ADV, HCV, HHV-1, HHV-2, and RSV immunoreactivity was negative in the four histological patterns described. Therefore, in DAD histological patterns of acute exacerbation of IPF and NSIP, we detected immunoreactivity for MV and CMV in alveolar epithelium in 38 and $10 \%$ of the cases, respectively. Alveolar epithelium infection by CMV was observed in $25 \%$ with OP patterns. The cases diagnosed only with the UIP and NSIP patterns had no viral infection detected by immunohistochemistry.

\section{Discussion}

In the present study, IHC indicated that in the DAD histological pattern found in AIP, in acute exacerbation of IPF and NSIP, immunoreactivity for MV and CMV in alveolar epithelium was found, respectively, in 25, 25, and $16 \%$ of the cases, whereas CMV was found in $25 \%$ of those with OP patterns. The histopathology of DAD is considered to represent end-stage phenomena in acutely behaving interstitial pneumonias, such as AIP and acute exacerbations of IPF and NSIP, although it should be stressed that DAD is more frequent in patients with IPF than in those with NSIP (16). In fact, several studies over a decade ago have suggested the role of viral infections in the pathogenesis of pulmonary fibrosis even if it is AE (17), particularly CMV (11). However, the different methodologies used in these studies to detect viral infection limits 
comparison of the real prevalence in IIPs. Another limitation is due to global differences in virus prevalence. For example, in Northern Brazil, Europe, and Northern America the spectrum of viral infections in children and the elderly is dominated by RSV (18). In Southern Africa, HSV has a higher prevalence (19).

Concerning MV detected in our study, in 2011, 1112 suspected cases of MV were reported by the Epidemiological Surveillance Center in São Paulo (20). Genetic characterization indicated that this virus had an identical $\mathrm{N}$ gene sequence and was a member of genotype D4, coincident with studies conducted by the World Health Organization (WHO), which show that the recent outbreaks in different parts of the world have been caused by this genotype (21). This fact - and our findings in the present study - strengthen the importance of continuing the close observation of measles in the São Paulo State and as a means of investigating whether new policies are needed to eliminate measles worldwide.

In the present study, our findings coincide with NCE but without demonstration of $\mathrm{CVI}$, showing the importance of the real interpretation of NCE identified on type II pneumocytes in cases where positive viral signals were not obtained. To our knowledge, this is the first study showing the importance of determining, in routine diagnosis, whether NCE is due to CMV or MV infection. This also confirms the well-known fact that immunohistochemical testing is more sensitive than the histological demonstration of CMV or MV inclusions. As recently postulated, virus-positive cells lack classic inclusions, and multiple viruses usually exhibit characteristic viral inclusions in infected host cells. However, there were some virus-positive cases in which no cytological changes suggestive of viral infection were identified on corresponding $\mathrm{HE}$ sections. Such findings have already been reported in lung, liver, and placenta for CMV and MV $(13,20-25)$. The precise relationship between the morphological changes and the degree of viral replication in infected host cells is still unclear $(25,26)$. However, it is believed that a large degree of full replication of CMV and $\mathrm{MV}$ causes cell enlargement with inclusions. It is presumed that, in liver (23), placenta (22), and lung (26), all infected cells do not always transcribe full viral antigens in some DAD/AIP cases. This finding supports the hypothesis that $\mathrm{IHC}$ is a more sensible method for detecting CMV- and MV-infected cells. The question arises whether or not the other cases that were positive for the viruses, but lacking inclusions, represented viral diseases. Moreover, although NCE may be the cause of malignancy, pathologists would benefit by reviewing other clinical information, particularly drugs that are known to be toxic to the lung, as well as by documenting other patterns of lung injury that may have been present. Changes such as concomitant increases in cytoplasm or the overall milieu of an inflammatory and reparative process usually point to cytotoxic effects (27). The distinction between these changes and viral infections may also be an issue unless a high threshold for the identification of viral inclusions is maintained. This is the reason why the immunohistochemical stain for viruses is so useful.

Interestingly, our cases were associated with DAD or acute exacerbation, suggesting that viral proliferation was not directly responsible for the development of IIPs, but was a harmless event that occurred after immunosuppression by treatment. It is also possible that the organism of these immunosuppressed patients hides the viral infection because they react differently from immunocompetent patients. Although the role of other viruses in the pathogenesis is still controversial, it has recently been hypothesized that DAD caused by CMV and MV is an immune-mediated event (28-30). Thus, regardless of the degree of CMV or MV replication in the lungs, these viruses can trigger DAD or influence the severity of concomitant pulmonary inflammation. In addition, CMV and MV infection may be important causes of death in patients with DAD who are immunosuppressed by treatment of the primary underlying diseases (31-34). However, because coexisting forms of pulmonary pathology are usually present, the role of CMV and MV in causing morbidity and mortality in DAD patients needs to be verified. Regardless of the low density of inclusion bodies, severe DAD was still documented. This observation may indicate that CMV and MV are common causes of severe diffuse lung damage in IIP patients. Thus, evidence of respiratory impairment would indicate treatment for CMV and MV in patients with IIPs.

Our results also suggest that a direct involvement of CMV and MV in DAD is likely, but this does not apply to the UIP and NSIP pattern without acute exacerbation. In fact, viral inclusions are more common in acute exacerbation of UIP $(4,5)$. The presence of viral inclusions in the lungs with interstitial inflammation, hyaline membranes, and giant cells is generally accepted as a diagnostic finding for CMV and MV pneumonia, respectively, despite the extent of virus-infected cells present. Thus, it is reasonable to consider that, in this study, at least 10 cases were viral pneumonitis, and DAD or hyaline membranes could be very strong markers of injury determined by viral infection. UIP and NSIP were the predominant histological patterns found, respectively, in 8 and 5 patients and were not associated with EBV, CMV, ADV, HCV, RSV, and HHV, thus suggesting that other agents, infectious or not, may also be associated with UIP and NSIP or the use of more sensitive and specific methods such as PCR (7-12). However, as indicated by Egan et al. (7), although PCR is considered to be sensitive, the application of $\mathrm{IHC}$ has a particular advantage in allowing localization of virus within the lung tissue. Together, these findings again emphasize the strong association between severe pulmonary damage and $\mathrm{CMV}$ or MV infections, establishing 
pathological criteria for treatment with potentially effective drugs.

We concluded that, in patients with IIP, CMV, and MV, infection might lead to death from a DAD pattern. For detection, IHC proved to be more effective than the histological demonstration of $\mathrm{CMV}$ or MV inclusions. These findings suggest that sensitive techniques, such

\section{References}

1. American Thoracic Society/European Respiratory Society International Multidisciplinary Consensus Classification of the Idiopathic Interstitial Pneumonias. Am J Respir Crit Care Med 2002; 165: 277-304, doi: 10.1164/ajrccm.165.2.ats01.

2. Monaghan H, Wells AU, Colby TV, du Bois RM, Hansell DM, Nicholson AG. Prognostic implications of histologic patterns in multiple surgical lung biopsies from patients with idiopathic interstitial pneumonias. Chest 2004; 125: 522526, doi: 10.1378/chest.125.2.522.

3. Raghu G, Collard HR, Egan JJ, Martinez FJ, Behr J, Brown KK, et al. An official ATS/ERS/JRS/ALAT statement: idiopathic pulmonary fibrosis: evidence-based guidelines for diagnosis and management. Am J Respir Crit Care Med 2011; 183: 788-824, doi: 10.1164/rccm.2009-040GL.

4. Churg A, Muller NL, Silva Cl, Wright JL. Acute exacerbation (acute lung injury of unknown cause) in UIP and other forms of fibrotic interstitial pneumonias. Am J Surg Pathol 2007; 31: 277-284, doi: 10.1097/01.pas.0000213341.70852.9d.

5. Collard HR, Moore BB, Flaherty KR, Brown KK, Kaner RJ, King TE Jr, et al. Acute exacerbations of idiopathic pulmonary fibrosis. Am J Respir Crit Care Med 2007; 176: 636-643, doi: 10.1164/rccm.200703-463PP.

6. Churg A, Wright JL, Tazelaar HD. Acute exacerbations of fibrotic interstitial lung disease. Histopathology 2011; 58: 525-530, doi: 10.1111/j.1365-2559.2010.03650.x.

7. Egan JJ, Stewart JP, Hasleton PS, Arrand JR, Carroll KB, Woodcock AA. Epstein-Barr virus replication within pulmonary epithelial cells in cryptogenic fibrosing alveolitis. Thorax 1995; 50: 1234-1239, doi: 10.1136/thx.50.12.1234.

8. Kuwano K, Nomoto Y, Kunitake R, Hagimoto N, Matsuba T, Nakanishi $Y$, et al. Detection of adenovirus E1A DNA in pulmonary fibrosis using nested polymerase chain reaction. Eur Respir J 1997; 10: 1445-1449, doi: 10.1183/09031936. 97.10071445.

9. Meliconi R, Andreone P, Fasano L, Galli S, Pacilli A, Miniero $\mathrm{R}$, et al. Incidence of hepatitis $\mathrm{C}$ virus infection in Italian patients with idiopathic pulmonary fibrosis. Thorax 1996; 51: 315-317, doi: 10.1136/thx.51.3.315.

10. Stewart JP, Egan JJ, Ross AJ, Kelly BG, Lok SS, Hasleton $\mathrm{PS}$, et al. The detection of Epstein-Barr virus DNA in lung tissue from patients with idiopathic pulmonary fibrosis. $\mathrm{Am} \mathrm{J}$ Respir Crit Care Med 1999; 159: 1336-1341, doi: 10.1164/ ajrccm.159.4.9807077.

11. Yonemaru $M$, Kasuga $I$, Kusumoto $H$, Kunisawa $A$, Kiyokawa $\mathrm{H}$, Kuwabara $\mathrm{S}$, et al. Elevation of antibodies to cytomegalovirus and other herpes viruses in pulmonary fibrosis. Eur Respir J 1997; 10: 2040-2045, doi: 10.1183/ 09031936.97.10092040.

12. Hayashi S, Hogg JC. Adenovirus infections and lung disease. Curr Opin Pharmacol 2007; 7: 237-243, doi: as IHC, should be combined with histological routine procedures.

\section{Acknowledgments}

Research supported by CNPq (\#300993/2010-2) and FAPESP (\#2010/09938-3). 10.1016/j.coph.2006.11.014.

13. Andrade ZR, Garippo AL, Saldiva PH, Capelozzi VL. Immunohistochemical and in situ detection of cytomegalovirus in lung autopsies of children immunocompromised by secondary interstitial pneumonia. Pathol Res Pract 2004 200: 25-32, doi: 10.1016/j.prp.2003.12.004.

14. Akira M, Hamada H, Sakatani M, Kobayashi C, Nishioka M, Yamamoto S. CT findings during phase of accelerated deterioration in patients with idiopathic pulmonary fibrosis. AJR Am J Roentgenol 1997; 168: 79-83, doi: 10.2214/ajr. 168.1.8976924.

15. Bubendorf L, Nocito A, Moch H, Sauter G. Tissue microarray (TMA) technology: miniaturized pathology archives for high-throughput in situ studies. J Pathol 2001; 195: 72-79, doi: 10.1002/path.893.

16. Kaarteenaho R, Kinnula VL. Diffuse alveolar damage: a common phenomenon in progressive interstitial lung disorders. Pulm Med 2011; 2011: 531302.

17. Vannella KM, Luckhardt TR, Wilke CA, van Dyk LF, Toews $\mathrm{GB}$, Moore BB. Latent herpesvirus infection augments experimental pulmonary fibrosis. Am J Respir Crit Care Med 2010; 181: 465-477, doi: 10.1164/rccm.200905-0798OC.

18. Bloom-Feshbach K, Alonso WJ, Charu V, Tamerius J, Simonsen L, Miller MA, et al. Latitudinal variations in seasonal activity of influenza and respiratory syncytial virus (RSV): a global comparative review. PLoS One 2013; 8: e54445, doi: 10.1371/journal.pone.0054445.

19. Dudek TE, Torres-Lopez E, Crumpacker C, Knipe DM. Evidence for differences in immunologic and pathogenesis properties of herpes simplex virus 2 strains from the United States and South Africa. J Infect Dis 2011; 203: 1434-1441, doi: 10.1093/infdis/jir047.

20. Oliveira MI, Figueiredo CA, Afonso AM, Santos FC, Lemos $\mathrm{XR}, \mathrm{Yu} A \mathrm{~L}$, et al. Resurgence of measles virus in São Paulo, Brazil. Rev Inst Med Trop São Paulo 2012; 54: 113-114, doi: 10.1590/S0036-46652012000200010.

21. Rota PA, Brown K, Mankertz A, Santibanez S, Shulga S, Muller $\mathrm{CP}$, et al. Global distribution of measles genotypes and measles molecular epidemiology. J Infect Dis 2011; 204 (Suppl 1): S514-S523, doi: 10.1093/infdis/jir118.

22. Muhlemann K, Miller RK, Metlay L, Menegus MA Cytomegalovirus infection of the human placenta: an immunocytochemical study. Hum Pathol 1992; 23: 12341237, doi: 10.1016/0046-8177(92)90290-J.

23. Theise ND, Conn M, Thung SN. Localization of cytomegalovirus antigens in liver allografts over time. Hum Pathol 1993; 24: 103-108, doi: 10.1016/0046-8177(93)90069-S.

24. Sata T, Kurata T, Aoyama Y, Sakaguchi M, Yamanouchi K, Takeda K. Analysis of viral antigens in giant cells of measles pneumonia by immunoperoxidase method. Virchows Arch $A$ 
Pathol Anat Histopathol 1986; 410: 133-138, doi: 10.1007/ BF00713517.

25. Ng-Bautista CL, Sedmak DD. Cytomegalovirus infection is associated with absence of alveolar epithelial cell HLA class II antigen expression. J Infect Dis 1995; 171: 39-44, doi: 10.1093/infdis/171.1.39.

26. Swoveland PT, Johnson KP. Enhancement of fluorescent antibody staining of viral antigens in formalin-fixed tissues by trypsin digestion. J Infect Dis 1979; 140: 758-764, doi: 10.1093/infdis/140.5.758.

27. Colby TV, Carrington CB. Interstitial lung disease. In: Thurlbeck WM, Churg AC (Editors), Pathology of the lung. 2nd edn. New York: Thieme; 1986. p 589-737.

28. Nakano $T$, Shimono $Y$, Sugiyama $K$, Nishihara $H$, Higashigawa M, Komada Y, et al. Clinical features of measles in immunocompromised children. Acta Paediatr Jpn 1996; 38: 212-217, doi: 10.1111/j.1442-200X.1996.tb03472.x.

29. Okamura A, Itakura O, Yoshioka M, Kubota M, Kikuta H, Kobayashi K. Unusual presentation of measles giant cell pneumonia in a patient with acquired immunodeficiency syndrome. Clin Infect Dis 2001; 32: E57-E58, doi: 10.1086/ 318499 .
30. Grundy JE, Shanley JD, Griffiths PD. Is cytomegalovirus interstitial pneumonitis in transplant recipients an immunopathological condition? Lancet 1987; 2: 996-999, doi: 10.1016/S0140-6736(87)92560-8.

31. Gutierrez EB, Zanetta DM, Saldiva PH, Capelozzi VL. Autopsy-proven determinants of death in HIV-infected patients treated for pulmonary tuberculosis in São Paulo, Brazil. Pathol Res Pract 2002; 198: 339-346, doi: 10.1078/ 0344-0338-00264.

32. Komatsuda A, Chubachi A, Miura AB. Virus-associated hemophagocytic syndrome due to measles accompanied by acute respiratory failure. Intern Med 1995; 34: 203-206, doi 10.2169/internalmedicine.34.203.

33. Siegel C, Johnston S, Adair S. Isolation of measles virus in primary rhesus monkey cells from a child with acute interstitial pneumonia who cytologically had giant-cell pneumonia without a rash. Am J Clin Pathol 1990; 94 464-469.

34. Gonzalez AC, Leon LA, Rafel E, Armenta GD, Salgado W, Guzman Zamudio JL. [Giant cell pneumonia. Complication in one case of acute lymphoblastic leukemia (author's transI)]. An Esp Pediatr 1980; 13: 1101-1104. 